\title{
Discovery of a diagnostic biomarker for colon cancer through proteomic profiling of small extracellular vesicles
}

\author{
Chan-Hyeong Lee ${ }^{1,2}$, Eun-Ju Im ${ }^{1,2}$, Pyong-Gon Moon ${ }^{1,2^{*}}$ and Moon-Chang Baek ${ }^{1,2^{*}}$
}

\begin{abstract}
Background: Small extracellular vesicles (small-EVs) are membranous vesicles that contain unique information regarding the condition of cells and contribute to the recruitment and reprogramming of components associated with the tumor environment. Therefore, many researchers have suggested that small-EV proteins are potential biomarkers for diseases such as cancer. Colon cancer (CC) is one of the most common causes of cancer-related deaths worldwide. Biomarkers such as carcinoembryonic antigen (CEA) show low sensitivity ( 40\%), and thus the demand for novel biomarkers for CC diagnosis is increasing.

Methods: In this study, we identified biomarkers for diagnosing CC through proteomic analysis of small-EVs from CC cell lines. These small-EVs were characterized by western blot analysis, nanoparticle tracking analysis, and transmission electron microscopy and analyzed using mass spectrometry.

Results: Five selected proteins were found to be upregulated in CC by western blot analysis. Among the candidate proteins, tetraspanin 1 (TSPAN1) was found to be upregulated in plasma EVs from CC patients compared to those from healthy controls (HCs) with $75.7 \%$ sensitivity.

Conclusions: These results suggest that TSPAN1 is a potent non-invasive biomarker for CC detection. Our experimental strategy provides useful insights into the identification of cancer-specific non-invasive biomarkers.
\end{abstract}

Keywords: Small extracellular vesicle, Proteomics, Colon cancer, Biomarker, Diagnosis

\section{Background}

Colon cancer (CC) is the third most common cause of cancer-related deaths worldwide and its mortality and incidence rates are increasing rapidly [1]. This disease mainly occurs in males and females 55-85 years of age; this age group accounts for approximately $80 \%$ of CC cases. Common risk factors for $\mathrm{CC}$ include obesity, family history, and physical inactivity [2-4]. CC is typically diagnosed by endoscopic biopsy and can be carried out by either sigmoidoscopy (as $>35 \%$ of tumors are in the rectosigmoid) or (preferably) total colonoscopy. [5]. However, these methods have limitations in terms of their convenience, risk, cost, and sensitivity [6]. Blood-based biomarkers can be analyzed easily, quickly, and economically, and therefore have the

\footnotetext{
*Correspondence: pyonggonmoon@gmail.com; mcbaek@knu.ac.kr ${ }^{1}$ Department of Molecular Medicine, CMRI, School of Medicine, Kyungpook National University, Daegu 41944, Republic of Korea
}

Full list of author information is available at the end of the article potential to greatly improve diagnostic efficiency. In 1965 , Dr. Joseph Gold discovered a protein that is normally found in the gastrointestinal tissue during fetal development in the blood of patients with colon cancer, which was named as carcinoembryonic antigen (CEA). This protein is now used as a biomarker for the diagnosis and prognosis of CC in hospitals [7]. However, most biomarkers for other cancers, including CEA, show limited sensitivity [8]. Thus, novel diagnostic biomarkers with high specificity and sensitivity are required for diagnosing $\mathrm{CC}$.

Extracellular vesicles (EVs) are membrane-bound vesicles secreted by a variety of cell types and are found in various body fluids including the blood, urine, breast milk, and malignant ascites [9]. These vesicles also contain oncogenic proteins [9], signaling molecules [10], lipids [11], mRNAs, and microRNAs [12] that reflect parental cell functions [13], and can be horizontally transferred to recipient cells to regulate their characterization [12].

(c) The Author(s). 2018 Open Access This article is distributed under the terms of the Creative Commons Attribution 4.0 International License (http://creativecommons.org/licenses/by/4.0/), which permits unrestricted use, distribution, and reproduction in any medium, provided you give appropriate credit to the original author(s) and the source, provide a link to the Creative Commons license, and indicate if changes were made. The Creative Commons Public Domain Dedication waiver (http://creativecommons.org/publicdomain/zero/1.0/) applies to the data made available in this article, unless otherwise stated. 
Among these, small extracellular vesicles (small-EVs), including exosomes, have been studied in various cancer types [13] and small-EV cargo contains more oncoproteins than large EVs [14].

Cancer cells secrete small-EVs containing oncogenic molecules into the extracellular environment, which play important roles in metastasis, angiogenesis, cancer cell proliferation, modulation of the tumor microenvironment, and immune suppression [15]. It has been suggested that cancer cell-derived small-EVs can be used as biomarkers for the diagnosis and prognosis of various cancers [16-18].

Recently, small-EVs isolated from cancer cells have been used to identify novel cancer-related biomarkers. Several studies were performed to identify molecular biomarkers in cancer cell-derived small-EVs [18-20]. However, most methods require a long time and large sample volume for small-EV isolation. To overcome these problems, we developed an enzyme-linked immunosorbent assay (ELISA) method to detect small-EV proteins without isolating the small-EVs. We examined whether this method can be used to diagnose early breast cancer; surface proteins (Del-1 and fibronectin) in small-EVs were detected in plasma obtained from patients with breast cancer with high sensitivity and specificity [21, 22]. The results suggest that an optimized ELISA method can detect surface proteins in small-EVs and that small-EV proteins can effectively distinguish between HCs and cancer patients.

In this study, we aimed to discover potential biomarkers in small-EVs through proteomic analysis for diagnosing $\mathrm{CC}$ and identified small-EVs from plasma for distinguishing between healthy controls ( $\mathrm{HCs}$ ) and $\mathrm{CC}$ patients without the need of an isolation step for small-EVs.

\section{Methods}

\section{Cell lines and cell culture}

HT-29 (ATCC ${ }^{\oplus}$ Number HTB-38 ${ }^{\mathrm{Tm}}$ ), HCT-116 (ATCC ${ }^{\bullet}$ Number CCL-247 ${ }^{\text {TM }}$ ) CC epithelial cells and CRL-1541 (ATCC ${ }^{\ominus} \mathrm{CRL}^{-1541^{\mathrm{TM}}}$ ) colon normal fibroblasts were cultured in Dulbecco's modified Eagle's medium (DMEM) supplemented with $10 \%$ EV-depleted fetal bovine serum (FBS) and 1\% antibiotic/antimycotic solution. Cells were obtained from the American Type Culture Collection (Manassas, VA, USA) and tested for mycoplasma contamination by PCR.

\section{Cell proliferation assay}

HT-29 and HCT-116 cells (50,000 cells/well) were seeded into 24-well plates in DMEM supplemented with $10 \%$ EV-depleted FBS and cultured to 90\% confluence. The medium was replaced with serum-free medium after 0,12 , 24, and $48 \mathrm{~h}$, and $0.5 \mathrm{mg} / \mathrm{mL}$ MTT (3-(4,5-dimethylthiazol-2yl)-2,5-diphenyltetrazolium bromide) solution was added to each well and incubated for $3 \mathrm{~h}$ at $37{ }^{\circ} \mathrm{C}$. Five hundred microliters of $100 \%$ isopropanol was added, $200 \mu \mathrm{L}$ of this mixture was transferred to 96-well plates, and absorbance was measured at $495 \mathrm{~nm}$.

\section{Small-EV purification}

Small-EVs were purified by differential centrifugation as described previously [23, 24]. Briefly, supernatants from CC cells were subjected to differential centrifugation at 300, 1500, 2500, and 100,000 $\times g$. Small-EVs $(100,000 \times g$ pellet) were resuspended in phosphate-buffered saline (PBS) for further analysis. As an alternative method for purification of small-EVs from plasma, the plasma was mixed with ExoQuick (System Biosciences, Mountain View, CA, USA). An optimized ExoQuick method was used as described in our previous study [25]. In the optimized ExoQuick method, after incubation at $4{ }^{\circ} \mathrm{C}$ for $30 \mathrm{~min}$, the samples were centrifuged at $1500 \times g$ for $30 \mathrm{~min}$, and the pellet was washed three times with PBS. These extra steps were required to reduce contamination with plasma proteins such as albumin. Small-EV proteins were quantified using the Pierce BCA Protein Assay kit (Rockford, IL, USA) after treatment with RIPA buffer (Cell Signaling Technology, Danvers, MA, USA).

\section{Transmission electron microscopy (TEM)}

Purified small-EVs were deposited onto pure carboncoated EM grids. After staining with $2 \%$ uranyl acetate, the grids were dried at $25{ }^{\circ} \mathrm{C}$ and visualized at $6000 \times$ and $10,000 \times$ magnification using the Hitachi H-7000 transmission electron microscope (Tokyo, Japan).

\section{Nanoparticle tracking analysis (NTA)}

The number of small-EVs was measured by NTA as described in our previous study [26]. Suspensions containing small-EVs from plasma or cell culture medium were analyzed using the Nano-Sight LM10 instrument (NanoSight, Wiltshire, UK). For this analysis, a monochromatic laser beam $(405 \mathrm{~nm})$ was applied to a dilute suspension of small-EVs. A video of 30- to 60-s duration was recorded at a rate of 30 frames/s, and small-EV movement was analyzed using NTA software version 2.2 (NanoSight). NTA post-acquisition settings were optimized and remained constant between samples, and each video was analyzed to estimate the concentration.

\section{Gel-assisted digestion}

Protein concentrations of small-EVs in HT-29, HCT-116, and CRL-1541 cells were determined using the BCA assay after cell lysis. Digestion was performed as described previously [24]. Briefly, $50 \mu \mathrm{g}$ protein was resuspended in $100 \mathrm{mM}$ triethylammonium bicarbonate (TEABC; $\mathrm{pH} 8$ ) containing $6 \mathrm{M}$ urea, $5 \mathrm{mM}$ EDTA, and 2\% SDS. The proteins were chemically denatured by adding 
$10 \mathrm{mM}$ dithiothreitol and incubating at $60{ }^{\circ} \mathrm{C}$ for $20 \mathrm{~min}$. Next, proteins were alkylated by adding $50 \mathrm{mM}$ iodoacetamide at room temperature and incubating for $20 \mathrm{~min}$. The protein solution was mixed with acrylamide/bisacrylamide solution (30\% v/v, 29:1) containing $10 \%(w / v)$ ammonium persulfate and tetramethylethylenediamine. The gel was cut into small pieces and washed three times with $50 \mathrm{mM}$ TEABC containing $50 \%$ acetonitrile $(\mathrm{ACN})$. Proteolytic digestion was performed using trypsin (protein:trypsin $=50: 1 \mathrm{w} / \mathrm{w}$ ) in $50 \mathrm{mM}$ TEABC and incubating overnight at $37^{\circ} \mathrm{C}$. The digested peptides were extracted from the gel by exchanging with two buffers: $0.1 \%$ formic acid (FA) in $50 \mathrm{mM}$ TEABC and $0.1 \% \mathrm{FA}$ in $50 \% \mathrm{ACN}$. The eluents were concentrated using a Speedvac and desalted using an HLB cartridge (Waters, Milford, MA, USA).

\section{Liquid chromatography-tandem mass spectrometry (LC- MS/MS) analysis}

Small-EVs were analyzed by LC-MS/MS as described previously [27]. One microgram of extracted peptide was analyzed by nano-ultra-high-performance LC (UPLC) (Waters) and tandem mass spectrometry using a Q-Tof Premier (Waters). Peptides were injected into a $2 \mathrm{~cm} \times$ $180 \mu \mathrm{m}$ trap column and resolved in a $25 \mathrm{~cm} \times 75 \mu \mathrm{m}$ nanoACQUITY C18 column (Waters) using the LC system. Mobile phase A was composed of water containing $0.1 \%$ FA and mobile B phase was $0.1 \%$ FA in ACN. The peptides were resolved using a gradient of $3-45 \%$ mobile phase B over $135 \mathrm{~min}$ at a flow rate of $300 \mathrm{~nL} / \mathrm{min}$. All samples were analyzed in triplicate. The method included a full sequential MS scan (m/z 150-1600, $0.6 \mathrm{~s})$ and five MS/MS scans (m/z 100-1990, $0.6 \mathrm{~s} / \mathrm{scan}$ ) for the five most intense ions present in the full-scan mass spectrum.

\section{Proteomic data processing and analysis}

For protein identification, MS raw data were converted into peak lists using MASCOT Distiller version 2.1 (Matrix Science, London, UK) with default settings. All MS/MS raw data were analyzed using MASCOT version 2.2.1 (Matrix Science) [27]. Mascot was used to search the Swiss-Prot database (release 2018_07) with human taxonomy. The database search against Mascot was performed with a fragment ion mass tolerance of $0.5 \mathrm{Da}$ and parent ion tolerance of $0.2 \mathrm{Da}$. Two missed cleavages were allowed for trypsin digestion. Carbamidomethylation of cysteine residues and oxidation of methionine residues were considered as variable modifications. To evaluate the false discovery rate (FDR) of protein identification, this analysis was repeated using identical search parameters and validation criteria against a randomized decoy database created by Mascot. Peptide identities were assigned if their Mascot ion scores corresponded to $p<0.05$. Proteins with more than 2 peptides were identified with confidence. FDRs with Mascot protein scores $>34(p<0.05)$ ranged below $2 \%$.

\section{Human plasma collection}

Human blood samples were obtained from 33 healthy donors and 46 patients with CC at Kyungpook National University Hospital (KNUH) and Chonbuk National University Hospital. All individuals provided informed consent for blood donation according to a protocol approved by the Institutional Review Board of KNUH. The blood samples of subjects from both groups were collected preoperatively in 9-mL vacutainers containing EDTA. The collected blood samples were centrifuged at $1500 \times g$ for $15 \mathrm{~min}$ to obtain plasma. These supernatants were stored at $-80{ }^{\circ} \mathrm{C}$ until use.

\section{Immunoblotting}

Proteins were resolved by SDS-PAGE, transferred onto nitrocellulose membranes, probed with each primary antibody, and incubated with horseradish peroxidaseconjugated secondary antibody. The blots were visualized with enhanced chemiluminescence detection reagents (Thermo Fisher Scientific, Waltham, MA, USA) and quantified using enhanced chemiluminescence hyperfilm (AGFA, Morstel, Belgium). The following primary antibodies were used against CD63 (ab68418, 1:1000), HSP70 (ab2787, 1:1000), CD9 (ab92726, 1:1000), Alix (ab56932, 1:1000), LGALS3BP (ab123921, 1:1000), SLC1A5 (ab58690, 1:1000), CLDN7 (ab53044, 1:1000), RAI3 (ab155557, 1:1000; all from Abcam, Cambridge, UK), and TSPAN1 (H00010103, 1:1000; Abnova, Taipei City, Taiwan).

\section{Elisa}

The method for detecting small-EV proteins has been described previously [21]. Anti-CD63 antibody (1:250)-coated 96-well plates were blocked with PBS containing 0.05\% Tween 20 (PBS-T) for $3 \mathrm{~h}$ at room temperature. Serial dilutions of mouse serum were prepared in PBS-T supplemented with $10 \%$ horse serum. The diluted solution was added to the plates in duplicates and incubated for $2 \mathrm{~h}$ at room temperature. After washing, the samples in the plates were reacted with the monoclonal anti-TSPAN1 antibody pre-incubated with a horseradish peroxidase-linked secondary antibody for $30 \mathrm{~min}$ and developed using $3,3^{\prime}, 5,5^{\prime}$-tetramethylbenzidine-containing peroxide. The reaction was stopped, and optical density values were measured at $450 \mathrm{~nm}$ using an automated iMark (Bio-Rad, Hercules, CA, USA).

\section{Statistical analysis}

Data are presented as the mean $\pm S D$, as indicated in each graph. The Student's $t$-test was used to evaluate the differences between means for normally distributed immunoblotting data. The significance of the ELISA results 


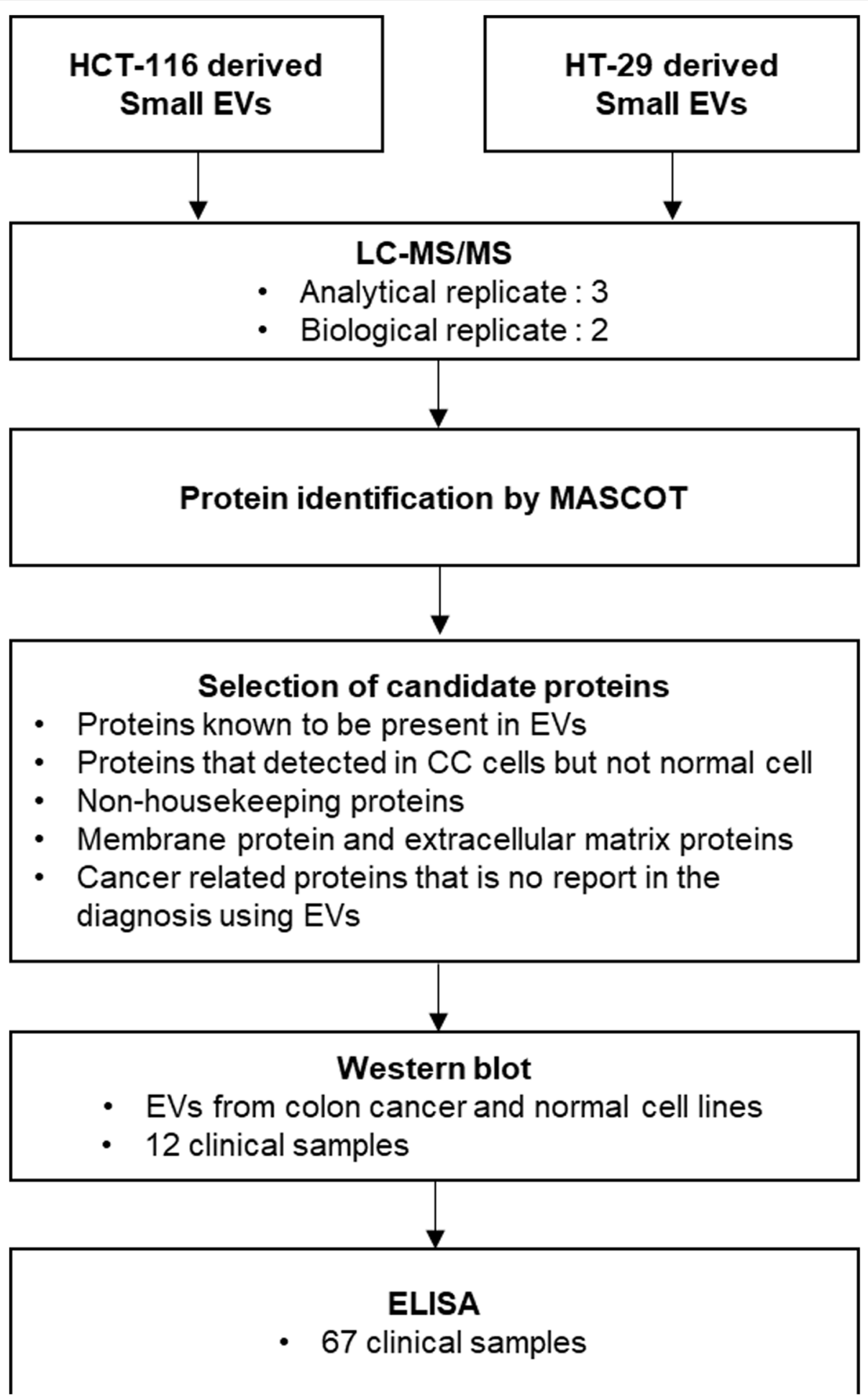

Fig. 1 Schematic representation of the workflow. Small-EV proteins from HT-29 and HCT-116 colon cancer cell lines were identified by proteomic analysis and candidate proteins predicted to be upregulated in colon cancer patients compared to the healthy controls were selected. Candidate proteins were verified by western blot analysis. Among these candidates, TSPAN1 was further validated by ELISA 
was analyzed using the Mann-Whitney test. All $p$-values were two-tailed, and $p$-values less than 0.05 were considered statistically significant. Receiver operating characteristic (ROC) curves were generated based on logistic regression from the ELISA data. Statistical analysis was performed using SPSS 22.0 for Windows (SPSS, Inc.,
Chicago, IL, USA). The area under ROC curve (AUC) calculation provided a convenient number. AUC $<0.5$ indicated that the test showed no difference between the two groups, while $0.5<\mathrm{AUC}<1.0$ indicated that the test yielded perfect differentiation between groups at ${ }^{*} p<0.05$, $* * 0<0.01,{ }^{* * * * *} p<0.001$, or ${ }^{* * * * *} p<0.0001$.
A

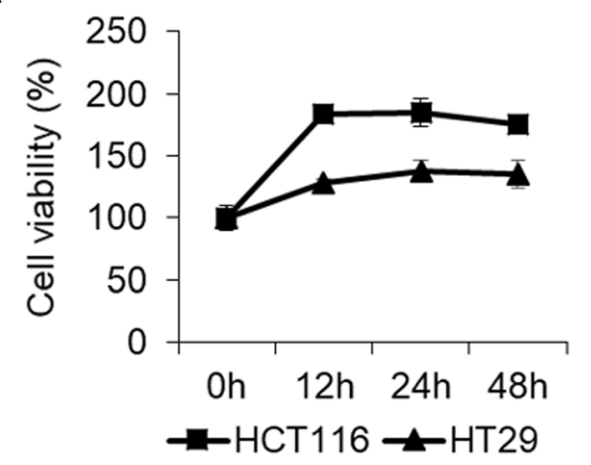

B

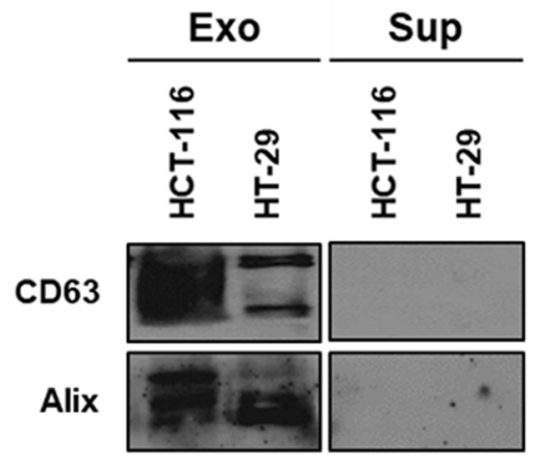

E

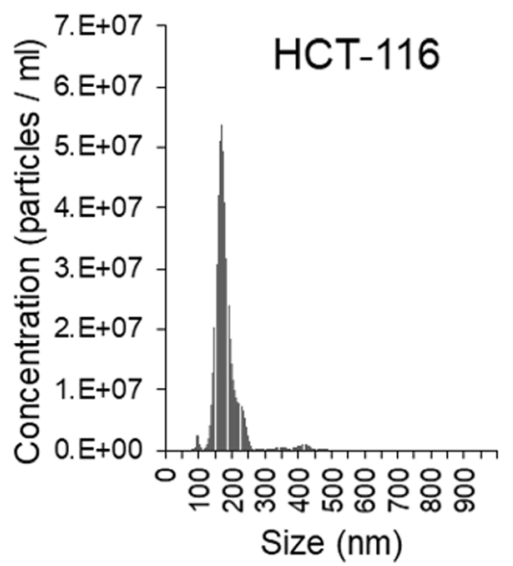

C

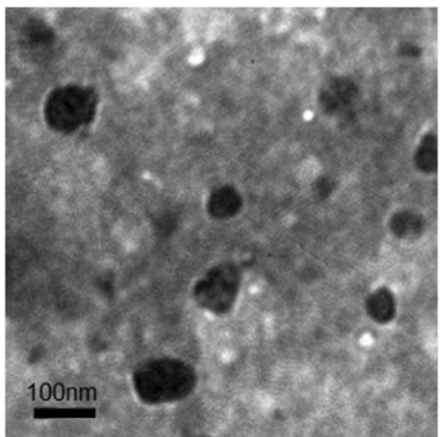

D

HCT-116

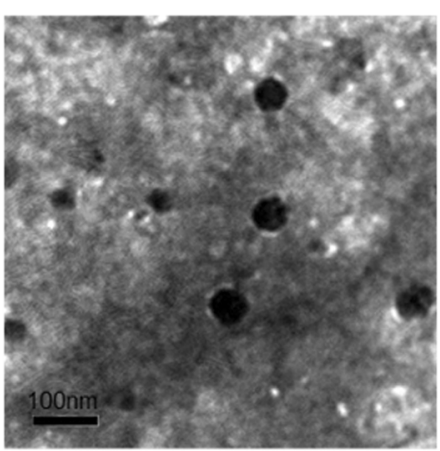

HT-29

F

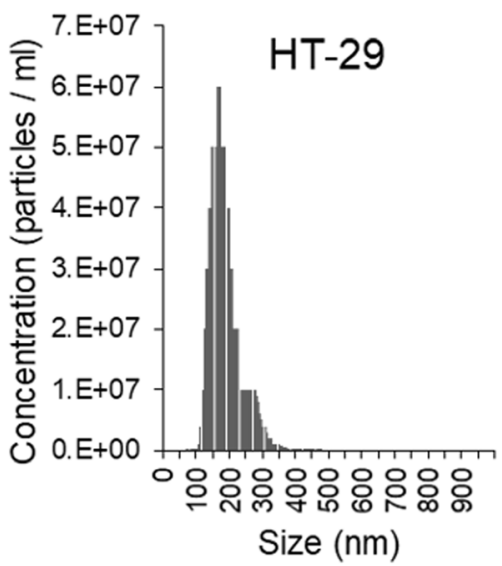

Fig. 2 Characterization of small-EVs isolated from HT-29 and HCT-116 colon cancer cells. a Viability of HT-29 and HCT-116 colon cancer cells following exposure to serum-free medium was measured using the MTT assay. b Small-EVs (10 $\mu \mathrm{g}$ protein) from HT-29 and HCT-116 cells were subjected to immunoblotting with antibodies against small-EV marker proteins, namely CD63 and Alix. c, d Small-EVs derived from HT-29 and HCT-116 cells were analyzed by transmission electron microscopy. All small-EV samples contained vesicles of variable sizes in the range of $70-200$ nm. Scale bar, $100 \mathrm{~nm}$. e, f Size distribution of small-EVs derived from two different colon cancer cell lines was measured using nanoparticle tracking analysis. The images shown are representative and the size of HT-29- and HCT-116-derived small-EVs is $184.2 \pm 46.5$ and $178.5 \pm 44.4 \mathrm{~nm}$, respectively $(n=3)$ 


\section{Results}

Isolation and characterization of small-EVs in colon cancer cell lines

To identify potential biomarkers for diagnosing colon cancer, HT-29 and HCT-116 human colon cancer cell lines were used in this study. A schematic workflow is shown in Fig. 1.
We analyzed cell viability at different incubation times under starvation conditions to optimize the incubation time and observed reduced cell viability at more than $24 \mathrm{~h}$ incubation (Fig. 2a). Isolated small-EVs were analyzed by western blotting, TEM, and NanoSight. Immunoblotting revealed that the small-EV fraction was highly enriched in small-EV markers such as CD63 and Alix (Fig. 2b). It also

A

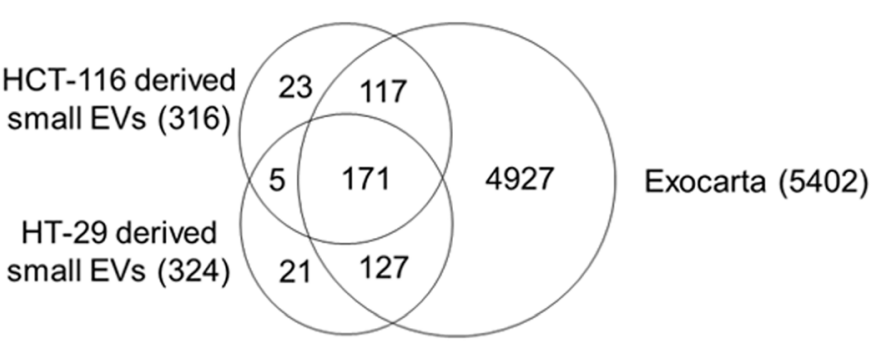

B

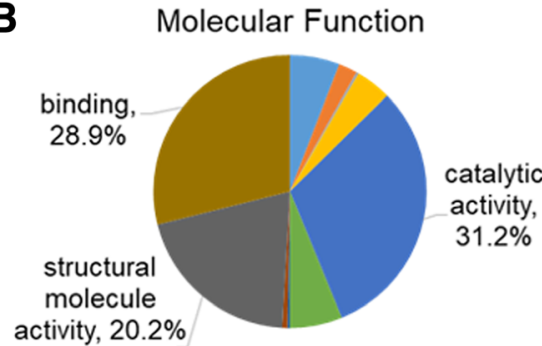

D

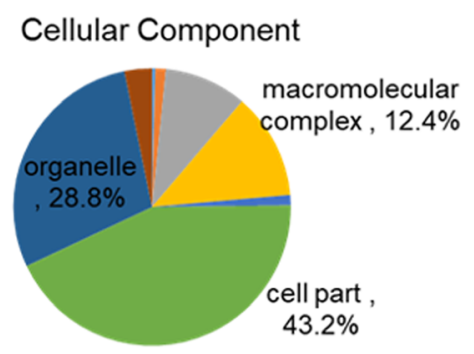

C Biological Process

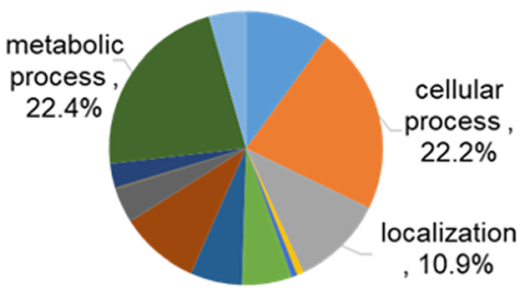

E

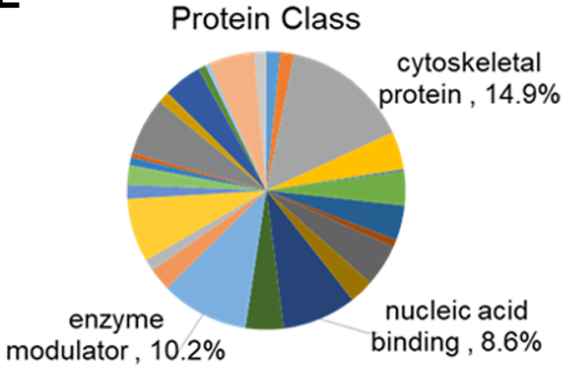

$\mathbf{F}$

Pathway

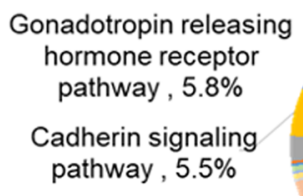

Cytoskeletal regulation by $\mathrm{Rho}$ GTPase , $5.8 \%$

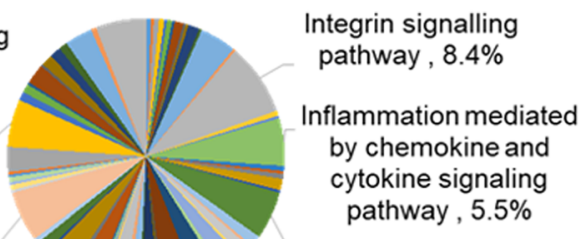

Huntington disease, $5.5 \%$

Fig. 3 Proteomic analysis of small-EVs from HCT-116 and HT-29 colon cancer cells. Search results were identified using MASCOT software. a At the cutoff settings, 464 human proteins were identified; of these, 176 proteins were observed to be common between the two cell lines. In total, 140 proteins were uniquely identified in the HCT-116-derived small-EVs and 148 proteins were identified in the HT-29-derived small-EVs. Among these, 415 proteins were present in the ExoCarta database. Identified proteins were segregated into various groups using PANTHER software. Gene ontology analysis for molecular function $\mathbf{b}$, biological process $\mathbf{c}$, cellular component $\mathbf{d}$, protein class $\mathbf{e}$, and pathway $\mathbf{f}$ of the proteins identified in small-EVs was performed using PANTHER software 


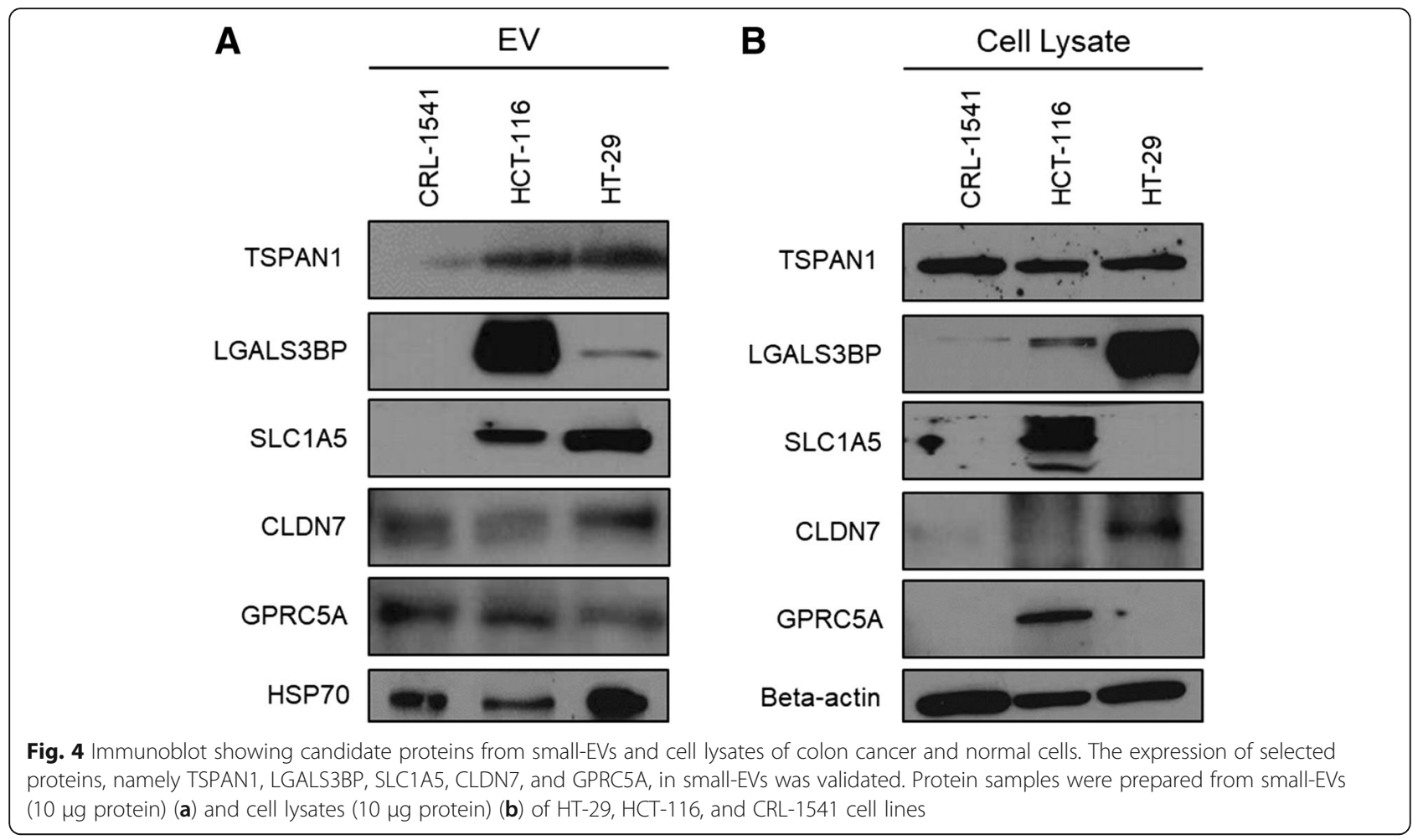

showed rounded morphology (Fig. 2c) and a size distribution (Fig. 2d) consistent with typical small-EVs.

\section{Identification of small-EVs from CC cells by proteomic analysis}

We performed proteomic analysis of small-EVs from CC cell lines by MS to identify small-EV proteins. CC cell-derived small-EVs were analyzed in two biological and three analytical replicates using nano-UPLC-MS/ MS. Small-EV proteins were searched in the Swiss-Prot database with human taxonomy using the MASCOT software. From among high-confidence peptide sequences with error rates $<5 \%$, we identified 316 and 324 proteins in HCT-116- and HT-29-derived small-EVs, respectively. This analysis showed that proteins with a peptide hit score $(\mathrm{PHS})>1$ were identified with high confidence by multiple peptides and overlapping gene names were removed. A total of 464 proteins were identified in CC cell-derived small-EVs with high confidence (PHS $>1$ ) (Fig. 3a; see Additional file 1: Table S1). Among these, 176 proteins were common between the two cell lines. We compared the identified proteins with the data in ExoCarta and observed that 90\% of the identified small-EV proteins were present in this database (Fig. 3a). For bioinformatics analysis, including prediction of the function, biological process, cellular components, localization, and pathways of the exosomal proteins, we used the Protein Analysis Through Evolutionary and Relationship (PANTHER) software. The identified proteins were associated with at least one annotation term. All searched proteins were categorized as having binding (36.3\%), catalytic (32.2\%), or structural (13.6\%) activities (Fig. 3b). The distribution of biological processes was as follows: cellular (29.1\%), metabolic (19\%), and cellular

Table 1 Information about clinical samples from patients with colon cancer and healthy controls

\begin{tabular}{|c|c|c|c|c|c|c|c|c|c|}
\hline \multirow{3}{*}{$\frac{\text { Detection method }}{\text { Clinical information }}$} & & \multicolumn{4}{|c|}{ Western blotting } & \multicolumn{4}{|l|}{ ELISA } \\
\hline & & \multirow{2}{*}{$\begin{array}{l}\text { \# of samples } \\
(n=12)\end{array}$} & \multirow[t]{2}{*}{ Age } & \multicolumn{2}{|c|}{ Sex } & \multirow{2}{*}{$\begin{array}{l}\text { \# of samples } \\
(n=67)\end{array}$} & \multirow[t]{2}{*}{ Age } & \multicolumn{2}{|l|}{ Sex } \\
\hline & & & & M & $\mathrm{F}$ & & & M & $F$ \\
\hline Healthy controls & & 3 & $46.3 \pm 10.9$ & 2 & 1 & 30 & $50.9 \pm 12.9$ & 15 & 15 \\
\hline \multirow[t]{4}{*}{ Colon cancer patients } & Stage 1 & 3 & $66 \pm 10.4$ & 2 & 1 & 10 & $68.7 \pm 10.8$ & 5 & 5 \\
\hline & Stage 2 & 3 & $64 \pm 4.6$ & 1 & 2 & 10 & $64 \pm 9.1$ & 5 & 5 \\
\hline & Stage 3 & 3 & $63.7 \pm 3.8$ & 2 & 1 & 10 & $67.4 \pm 7.1$ & 5 & 5 \\
\hline & Stage 4 & & & & & 7 & $63.4 \pm 12.4$ & 4 & 3 \\
\hline
\end{tabular}


component organization or biogenesis (12.2\%) (Fig. 3c). As shown in cellular components, $35.9 \%$ proteins were predicted to be localized in the cytoplasm, $24.5 \%$ in organelles, and $17.5 \%$ in macromolecular complexes (Fig. 3d). The distribution by protein class was as follows: enzyme modulator (15.9\%), nucleic acid-binding (13.2\%), and cytoskeletal protein (12.1\%) (Fig. 3e). Signaling pathway analysis of the identified proteins confirmed their associations with a variety of pathways (Fig. 3f).

Selection of candidate proteins for diagnosis of colon cancer From the identified proteins, we selected candidate proteins to identify CC-specific proteins. The filtration step to select the candidate proteins is shown in Fig. 1. A total of 464 small-EV proteins were identified in the HCT-116 and HT-29 cell-derived small-EVs by mass spectrometry. Among these, 415 proteins known to be present in EVs were selected. Proteins detected in HCT-116- and HT-29-derived small-EVs, but not in small-EVs from CRL-1541 or normal colon cells (see Additional file 2: Table S2), were selected (352). Next, we excluded housekeeping proteins such as actin, myosin, and ribosomal proteins (231). Extracellular matrix proteins and outer membrane proteins were selected to detect small-EVs in the plasma without a purification step (114). Finally, we selected the following five proteins based on previous studies: TSPAN1, galectin-3-binding protein (LGALS3BP), neutral amino acid transporter $\mathrm{B}(0)$ (SLC1A5), claudin 7 (CLDN7), and retinoic acid-induced protein 3 (GPRC5A).

\section{Expression of candidate proteins in HT-29- and HCT-116- derived small-EVs}

We performed immunoblotting analysis to detect candidate proteins in $\mathrm{CC}$ cells and confirm whether these proteins observed in the proteomic analysis were truly expressed in the small-EVs isolated from CC cells. CRL-1541 cells were analyzed and compared with CC cells. HSP70 was used as a known small-EV marker and $\beta$-actin was used as a cytosol marker for cell lysates. All candidate proteins were distinctly detected in CC cell-derived small-EVs (Fig. 4). Among these, expression of three proteins (SLC1A5, LGALS3BP, and TSPAN1) was increased in small-EVs isolated from CC cell lines.

\section{Expression of candidate proteins in the plasma of CC patients analyzed by immunoblotting assay}

To verify the levels of selected proteins in clinical samples, we used human plasma from HCs and patients with colon cancer. Detailed data related to clinical sample characteristics are provided in Table 1. After isolating small-EVs from the plasma, the selected proteins in small-EVs isolated from $\mathrm{HC}(n=3)$ and CC patients $(n=9)$ (Fig. $5 \mathrm{a})$ were verified

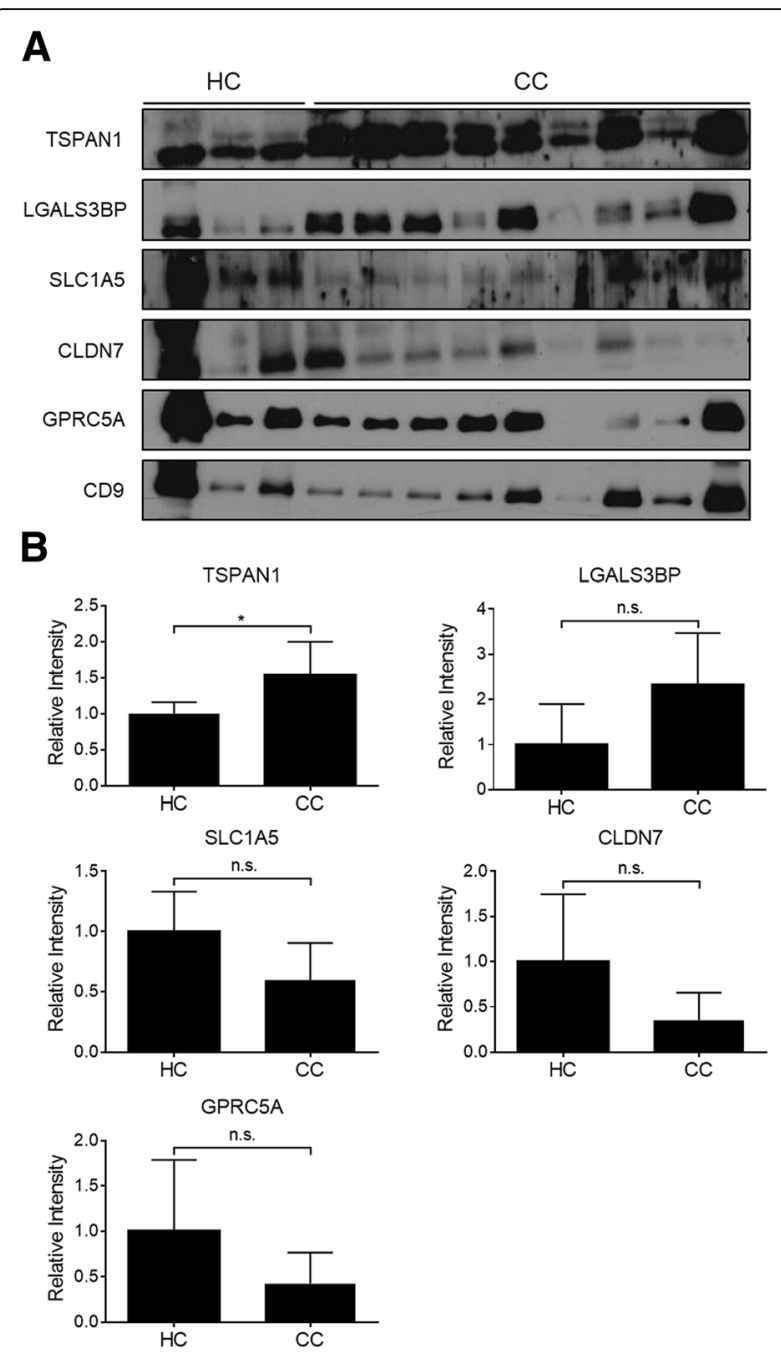

Fig. 5 Expression of candidate proteins in plasma-derived small-EVs. a Western blotting was performed to determine the expression level of five potential colon cancer markers and CD9 (a positive-control small-EV marker) in plasma-derived small-EVs $(4 \mu \mathrm{L})$ obtained from colon cancer patients (CC, $n=9)$ and healthy controls ( $H C, n=3)$. b Expression level and significance were validated. Significance was analyzed using the Student's $t$-test. ${ }^{*} p<0.05$

by immunoblotting. Relative expression of the candidate biomarker proteins is shown in Fig. $5 \mathrm{~b}$ in $\mathrm{HC}$ vs $\mathrm{CC}$ patients. TSPAN1 expression ( $\mathrm{HC}: \mathrm{CC}=1: 1.5, p=0.013$ ) was significantly higher in small-EVs isolated from the plasma of CC patients than in those of $\mathrm{HC}$ patients; however, LGALS3BP (HC:CC = 1:2.3, $p>0.05$ ), SLC1A5 (HC:CC = 1:0.6, $p>0.05$ ), CLDN7 (HC:CC=1:0.4, $p>0.05$ ), and GPRC5A ( $\mathrm{HC}: \mathrm{CC}=1: 0.4, p>0.05)$ expression was not significantly different between the two groups (Fig. 5b).

Diagnostic value of TSPAN1-positive small-EVs in plasma from CC patients analyzed using ELISA

We validated TSPAN1 in small-EVs from HCs $(n=30)$ and CC patients $(n=37)$ by ELISA. TSPAN1-positive 
small-EVs in plasma were captured using a coated anti-CD63 antibody and detected using an anti-TSPAN1 antibody (Fig. 6a). Our previous studies showed that this method was effective for detecting small-EVs [31, 32]. These results indicated that the TSPAN1 level was significantly higher in CC patients than in HCs (Fig. 6b). Additionally, ROC curves were generated using ELISA results to describe diagnostic performance (Fig. 6c), and AUC was determined for TSPAN1. Fig. 6 shows that the AUC of TSPAN1 for differentiating between CC patients and $\mathrm{HCs}$ was $0.828(95 \% \mathrm{CI}=0.73-0.926)$, with a sensitivity of $75.7 \%$ and specificity of $66.7 \%$.

\section{Discussion}

Small-EVs are informative vesicles that reflect the parent cell's physiological state and contain nucleic acids and proteins. Therefore, we focused on identifying biomarkers for diagnosing $\mathrm{CC}$ by analyzing small-EV proteins. In this study, we investigated cancer-related proteins from HCT-116 and HT-29 CC cell-derived small-EVs by proteomic analysis to identify diagnostic biomarkers for CC. By profiling of small-EVs from two
CC cell lines, 464 proteins were identified. Among these, approximately $90 \%$ were reported in the ExoCarta database [28]. Assignment of molecular function, biological process, cellular component, localization, and pathways revealed similar patterns to those observed in our previous studies [26]. This also corresponded with the current hypothesis for small-EV formation [29]. Figure 1 shows our strategy for selecting a diagnostic marker. We selected biomarker candidates that were cancer-related proteins in small-EVs produced by and exposed on the outer surface of cancer cells.

TSPAN1 is expressed in normal human tissues and carcinomas. Recently, TSPAN1 was also reported as a cancer-related protein $[30,31]$. In several studies, overexpression of TSPAN1 was observed in liver cancer, prostate cancer, and gastric carcinoma. TSPAN1 plays a role in cell mitosis and causes abnormal cell differentiation. It was detected by RT-PCR in cervical cancer, lung cancer, squamous carcinoma, colon cancer, and breast cancer cells [30]. Wollscheid et al. evaluated TSPAN1 mRNA levels by RT-PCR and TSPAN1 protein levels by immunohistochemistry in cervical cancer and found that

A

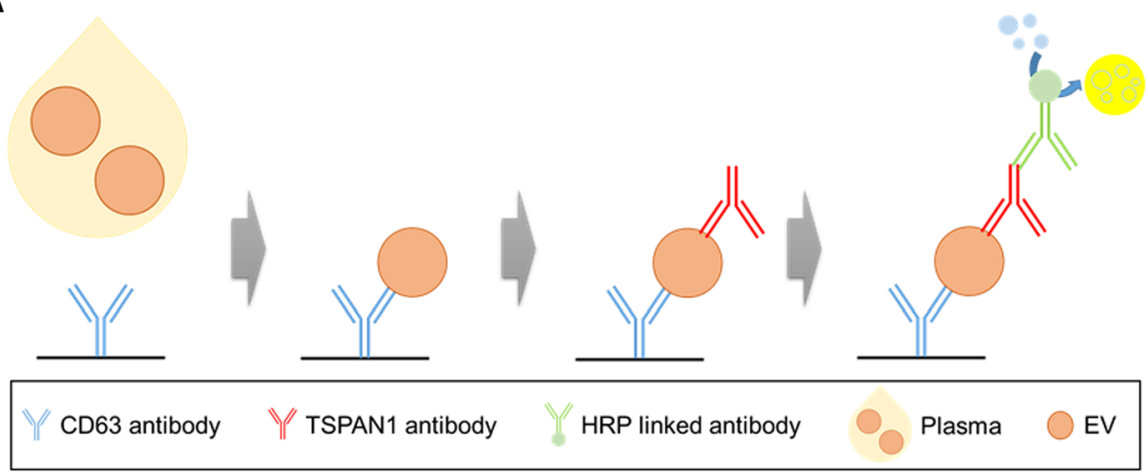

B

C
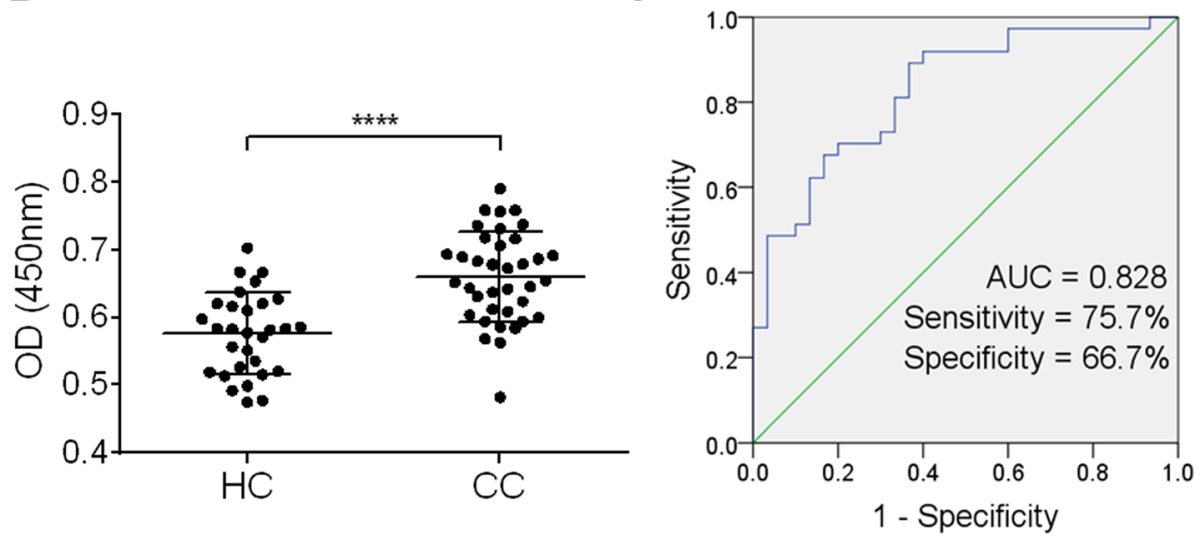

Fig. 6 Diagnostic value of TSPAN1-positive EVs in plasma. a TSPAN1-positive small-EVs in plasma were captured using the coated anti-CD63 antibody and detected using the anti-TSPAN1 antibody. $\mathbf{b}$ TSPAN1-positive small-EVs were validated in the plasma of colon cancer patients $(C C, n=37)$ and healthy controls $(H C, n=30)$. Significance was analyzed using the Mann-Whitney test. ${ }^{* * *} p<0.0001$. c ROCs for differentiating between CC patients and HCs were analyzed. The AUC, sensitivity, and specificity for TSPAN1 are shown 
the gene was expressed in grade III cervical intraepithelial neoplasia, cervical squamous cell carcinoma, and adenocarcinoma, particularly in all undifferentiated cervical carcinomas and adenocarcinomas [31]. They observed that TSPAN1 gene expression correlated with cell proliferation and suggested that it may be useful as a marker for cervical cancer prognosis.

In this study, a new strategy was established for identifying small-EV markers for $\mathrm{CC}$ diagnosis. However, further studies are required to evaluate their diagnostic value in clinical scenarios and elucidate their biological role(s) in cancer progression and metastasis. We performed western blotting to confirm the expression of candidate proteins in small-EVs from CC cells and normal colon cells and confirmed that SLC1A5 and LGALS3BP were differentially expressed between small-EVs and cells. However, previous studies reported that specific sorting of small-EV proteins can lead to changes in their composition [32, 33]. Expression levels of candidate proteins in small-EVs from the plasma of CC patients were confirmed by western blotting. We isolated small-EVs using ExoQuick solution. This method was fast and isolated small-EVs using only a small volume of plasma. However, recent studies reported that in current precipitation protocols, such as ExoQuick, small-EVs from cells and plasma were co-isolated with serum proteins [34]. Therefore, we used an optimized ExoQuick method to eliminate contaminants, such as albumin [25]. Recently, efforts have been undertaken to isolate small-EVs using more effective methods such as size-exclusion chromatography and tangential flow filtration $[34,35]$. Western blot analysis of 12 clinical samples revealed that TSPAN1 is a potent biomarker for CC diagnosis. Notably, the expression level of TSPAN1 was significantly higher in CC patients than in HCs. This result was validated by ELISA for 67 clinical samples.

TSPAN1-positive EVs showed diagnostic potential for CC with high sensitivity (75.7\%). In contrast, the CC biomarkers CEA and CA19-9 are currently adjunctively used for diagnosis and monitoring because of their low sensitivity [36, 37]. These results suggest that using TSPAN1-positive EVs or their combination with CEA or CA19-9 can increase the efficacy of diagnosis and that this strategy for discovering small-EV markers for cancer diagnosis is effective.

\section{Conclusions}

In conclusion, this study demonstrated that TSPAN1 was abundantly present in small-EVs from two CC cell lines. Further, analysis of small-EV proteins can be beneficial for identifying biofluid-based biomarkers for cancer diagnosis. Because cancer-derived small-EVs are found in body fluids such as the plasma and serum, TSPAN1 can act as a small-EV biomarker for advanced CC diagnosis.

\section{Additional files}

Additional file 1: Table S1. Protein list of small-EVs from HCT-116 and HT-29 CC cell lines. (XLSX 45 kb)

Additional file 2: Table S2. Protein list of small-EVs from CRL1541 normal colon cell line. (XLSX 14 kb)

\section{Abbreviations \\ AUC: Area under ROC curve; CC: Colon cancer; CEA: Carcinoembryonic antigen; CLDN7: Claudin-7; ELISA: Enzyme-linked immunosorbent assay; FDR: False discovery rate; GPRC5A: Retinoic acid-induced protein 3; HC: Healthy control; IPI: International Protein Index; LGALS3BP: Galectin-3- binding protein; NTA: Nanoparticle tracking analysis; PANTHER: Protein Analysis Through Evolutionary and Relationship; ROC: Receiver operating characteristic; SLC1A5: Neutral amino acid transporter B(0); Small-EV: Small extracellular vesicle; TEM: Transmission electron microscope; \\ TSPAN1: Tetraspanin-1}

\section{Acknowledgments}

The biospecimens for this study were provided by the National Biobank of Korea-Kyungpook National University Hospital and Chonbuk National University Hospital, which is supported by the Ministry of Health, Welfare and Affairs. All materials procured from the National Biobank of Korea-Kyungpook National University Hospital and Chonbuk National University Hospital were obtained under the institutional review board (IRB)-approved protocols.

\section{Funding}

This research was supported by the Bio \& Medical Technology Development Program of the National Research Foundation (NRF) funded by the Ministry of Science \& ICT (2017M3A9G 80833382). The funder was not involved in the design of the study and collection, analysis, and interpretation of data and in writing the manuscript.

\section{Availability of data and materials}

The datasets used and/or analyzed during the current study are available from the corresponding author on reasonable request.

\section{Authors' contributions}

CHL, EJI, PGM, and MCB participated in study design, data analysis, and preparation of the manuscript. CHL and PGM performed the experiments. All authors read and approved the final manuscript.

\section{Ethics approval and consent to participate}

This research was reviewed and approved by the Institutional Review Board of the Kyungpook National University (Korea) (KNUH 201411043003). All specimens were obtained from patients with written informed consent. The cell lines used in the study do not require ethic approval.

\section{Consent for publication}

Not applicable.

\section{Competing interests}

The authors declare that they have no competing interests.

\section{Publisher's Note}

Springer Nature remains neutral with regard to jurisdictional claims in published maps and institutional affiliations.

\section{Author details}

'Department of Molecular Medicine, CMRI, School of Medicine, Kyungpook National University, Daegu 41944, Republic of Korea. ${ }^{2}$ Exosome Convergence Research Center (ECRC), School of Medicine, Kyungpook National University, Daegu 41944, Republic of Korea. 
Received: 26 January 2018 Accepted: 15 October 2018

Published online: 01 November 2018

\section{References}

1. Ferlay J, Shin HR, Bray F, Forman D, Mathers C, Parkin DM. Estimates of worldwide burden of cancer in 2008: GLOBOCAN 2008. Int J Cancer. 2010; 127(12):2893-917.

2. Boyle T, Keegel T, Bull F, Heyworth J, Fritschi L. Physical activity and risks of proximal and distal colon cancers: a systematic review and meta-analysis. J Natl Cancer Inst. 2012;104(20):1548-61.

3. Brandstedt J, Wangefjord S, Nodin B, Gaber A, Manjer J, Jirstrom K. Gender anthropometric factors and risk of colorectal cancer with particular reference to tumour location and TNM stage: a cohort study. Biol Sex Differ. 2012;3(1):23.

4. Inger DB. Colorectal cancer screening. Prim Care. 1999;26(1):179-87.

5. Labianca R, Nordlinger B, Beretta GD, Mosconi S, Mandalà M, Cervantes A, Arnold D, ESMO Guidelines Working Group. Early colon cancer: ESMO clinical practice guidelines for diagnosis, treatment and follow-up. Ann Oncol. 2013;24:vi64-72

6. Gold P, Freedman SO. Demonstration of tumor-specific antigens in human colonic Carcinomata by immunological tolerance and absorption techniques. J Exp Med. 1965;121:439-62

7. Gupta S, Sussman DA, Doubeni CA, Anderson DS, Day L, Deshpande AR, Elmunzer BJ, Laiyemo AO, Mendez J, Somsouk M, et al. Challenges and possible solutions to colorectal cancer screening for the underserved. J Natl Cancer Inst. 2014:106(4):dju032.

8. Polanski M, Anderson NL. A list of candidate cancer biomarkers for targeted proteomics. Biomark Insights. 2007;1:1-48.

9. Thery C, Zitvogel L, Amigorena S. Exosomes: composition, biogenesis and function. Nat Rev Immunol. 2002;2(8):569-79.

10. Putz U, Howitt J, Doan A, Goh CP, Low LH, Silke J, Tan SS. The tumor suppressor PTEN is exported in exosomes and has phosphatase activity in recipient cells. Sci Signal. 2012;5(243):ra70.

11. Record M, Subra C, Silvente-Poirot S, Poirot M. Exosomes as intercellular signalosomes and pharmacological effectors. Biochem Pharmacol. 2011; 81(10):1171-82.

12. Valadi H, Ekstrom K, Bossios A, Sjostrand M, Lee JJ, Lotvall JO. Exosomemediated transfer of mRNAs and microRNAs is a novel mechanism of genetic exchange between cells. Nat Cell Biol. 2007;9(6):654-9.

13. Azmi AS, Bao B, Sarkar FH. Exosomes in cancer development, metastasis, and drug resistance: a comprehensive review. Cancer Metastasis Rev. 2013; 32(3-4):623-42.

14. Keerthikumar S, Gangoda L, Liem M, Fonseka P, Atukorala I, Ozcitti C, Mechler A, Adda CG, Ang CS, Mathivanan S. Proteogenomic analysis reveals exosomes are more oncogenic than ectosomes. Oncotarget. 2015;6(17): 15375-96.

15. Tickner JA, Urquhart AJ, Stephenson SA, Richard DJ, O'Byrne KJ. Functions and therapeutic roles of exosomes in cancer. Front Oncol. 2014:4:127.

16. D'Souza-Schorey C, Clancy JW. Tumor-derived microvesicles: shedding light on novel microenvironment modulators and prospective cancer biomarkers. Genes Dev. 2012;26(12):1287-99.

17. Moon PG, You S, Lee JE, Hwang D, Baek MC. Urinary exosomes and proteomics. Mass Spectrom Rev. 2011;30(6):1185-202.

18. Melo SA, Luecke LB, Kahlert C, Fernandez AF, Gammon ST, Kaye J, LeBleu VS, Mittendorf EA, Weitz J, Rahbari N, et al. Glypican-1 identifies cancer exosomes and detects early pancreatic cancer. Nature. 2015;523(7559):177-82.

19. de Wit M, Kant H, Piersma SR, Pham TV, Mongera S, van Berkel MP, Boven E, Ponten F, Meijer GA, Jimenez CR, et al. Colorectal cancer candidate biomarkers identified by tissue secretome proteome profiling. J Proteome. 2014:99:26-39.

20. Ogata-Kawata H, Izumiya M, Kurioka D, Honma Y, Yamada Y, Furuta K, Gunji T, Ohta H, Okamoto $\mathrm{H}$, Sonoda $\mathrm{H}$, et al. Circulating exosomal microRNAs as biomarkers of colon cancer. PLoS One. 2014;9(4):e92921.

21. Moon PG, Lee JE, Cho YE, Lee SJ, Jung JH, Chae YS, Bae HI, Kim YB, Kim IS, Park HY, et al. Identification of developmental endothelial Locus-1 on circulating extracellular vesicles as a novel biomarker for early breast Cancer detection. Clin Cancer Res. 2016;22(7):1757-66.

22. Moon PG, Lee JE, Cho YE, Lee SJ, Chae YS, Jung JH, Kim IS, Park HY, Baek MC. Fibronectin on circulating extracellular vesicles as a liquid biopsy to detect breast cancer. Oncotarget. 2016;7(26):40189-99.
23. Thery C, Amigorena S, Raposo G, Clayton A. Isolation and characterization of exosomes from cell culture supernatants and biological fluids. Curr Protoc Cell Biol. 2006; Chapter 3(Unit 3):22.

24. Cho YE, Singh TS, Lee HC, Moon PG, Lee JE, Lee MH, Choi EC, Chen YJ, Kim SH, Baek MC. In-depth identification of pathways related to cisplatin-induced hepatotoxicity through an integrative method based on an informaticsassisted label-free protein quantitation and microarray gene expression approach. Mol Cell Proteomics. 2012;11(1):M111 010884.

25. Cho YE, Im EJ, Moon PG, Mezey E, Song BJ, Baek MC. Increased liver-specific proteins in circulating extracellular vesicles as potential biomarkers for drug- and alcohol-induced liver injury. PLoS One. 2017;12(2):e0172463.

26. Lee JE, Moon PG, Cho YE, Kim YB, Kim IS, Park H, Baek MC. Identification of EDIL3 on extracellular vesicles involved in breast cancer cell invasion. J Proteome. 2016;131:17-28.

27. Moon PG, Lee JE, You S, Kim TK, Cho JH, Kim IS, Kwon TH, Kim CD, Park SH, Hwang $D$, et al. Proteomic analysis of urinary exosomes from patients of early IgA nephropathy and thin basement membrane nephropathy. Proteomics. 2011;11(12):2459-75.

28. Mathivanan S, Simpson RJ. ExoCarta: a compendium of exosomal proteins and RNA. Proteomics. 2009:9(21):4997-5000.

29. Duijvesz D, Luider T, Bangma $\mathrm{CH}$, Jenster $\mathrm{G}$. Exosomes as biomarker treasure chests for prostate cancer. Eur Urol. 2011;59(5):823-31.

30. Serru V, Dessen P, Boucheix C, Rubinstein E. Sequence and expression of seven new tetraspans. Biochim Biophys Acta. 2000;1478(1):159-63.

31. Wollscheid V, Kuhne-Heid R, Stein I, Jansen L, Kollner S, Schneider A, Durst M. Identification of a new proliferation-associated protein NET-1/C4.8 characteristic for a subset of high-grade cervical intraepithelial neoplasia and cervical carcinomas. Int J Cancer. 2002;99(6):771-5.

32. Ohshima K, Kanto K, Hatakeyama K, Ide T, Wakabayashi-Nakao K, Watanabe Y, Sakura N, Terashima M, Yamaguchi K, Mochizuki T. Exosome-mediated extracellular release of polyadenylate-binding protein 1 in human metastatic duodenal cancer cells. Proteomics. 2014;14(20):2297-306.

33. Hoshino A, Costa-Silva B, Shen TL, Rodrigues G, Hashimoto A, Tesic Mark M, Molina H, Kohsaka S, Di Giannatale A, Ceder S, et al. Tumour exosome integrins determine organotropic metastasis. Nature. 2015:527(7578):329-35.

34. Lobb RJ, Becker M, Wen SW, Wong CS, Wiegmans AP, Leimgruber A, Moller A. Optimized exosome isolation protocol for cell culture supernatant and human plasma. J Extracell Vesicles. 2015;4:27031.

35. Heinemann ML, IImer M, Silva LP, Hawke DH, Recio A, Vorontsova MA, Alt E, Vykoukal J. Benchtop isolation and characterization of functional exosomes by sequential filtration. J Chromatogr A. 2014;1371:125-35.

36. Carpelan-Holmstrom MA, Haglund CH, Roberts PJ. Differences in serum tumor markers between colon and rectal cancer. Comparison of CA 242 and carcinoembryonic antigen. Dis Colon Rectum. 1996:39(7):799-805.

37. Nakayama T, Watanabe M, Teramoto T, Kitajima M. CA19-9 as a predictor of recurrence in patients with colorectal cancer. J Surg Oncol. 1997:66(4):238-43.

\section{Ready to submit your research? Choose BMC and benefit from:}

- fast, convenient online submission

- thorough peer review by experienced researchers in your field

- rapid publication on acceptance

- support for research data, including large and complex data types

- gold Open Access which fosters wider collaboration and increased citations

- maximum visibility for your research: over $100 \mathrm{M}$ website views per year

At $\mathrm{BMC}$, research is always in progress.

Learn more biomedcentral.com/submissions 